Retos, $\mathrm{n}^{\circ} 13$, vol.VII, 2017

\title{
El inventario como determinante en la rentabilidad de las distribuidoras farmacéuticas
}

\section{The Inventory as a determinant in the profitability of pharmaceutical distributors}

\author{
Luis Asencio Cristóbal \\ Universidad de Guayaquil-Ecuador \\ luis.asencioc@ug.edu.ec \\ orcid.org/0000-0003-3059-6389 \\ Edwin González Ascencio \\ Universidad de Guayaquil-Ecuador \\ edwin.gonzaleza@ug.edu.ec \\ orcid.org/0000-0002-0652-954 I \\ Mariana Lozano Robles \\ Universidad de Guayaquil-Ecuador \\ mariana.Iozanoro@ug.edu.ec \\ orcid.org/0000-0003-0876-2184
}

\section{Resumen}

En el mundo competitivo en que se desenvuelven los negocios, es necesario desarrollar mecanismos de control interno que permitan a las empresas reducir sus costos para obtener mayor utilidad. El presente trabajo tiene por objetivo analizar el control de inventarios, contextualizado en el sector de distribución farmacéutica de la provincia de Guayas-Ecuador, a fin de determinar su incidencia en los costos y en la rentabilidad de las empresas. La metodología a seguir para realizar un diagnóstico contable a profundidad, se sustenta en métodos y técnicas científicas (observación, entrevistas y encuestas) aplicadas en una empresa distribuidora farmacéutica, en la esfera del manejo de inventarios, siendo esta investigación exploratoria y descriptiva. Los resultados obtenidos, presentan los principales inconvenientes del control de inventarios en la distribución farmacéutica, que se centran en la carencia de mecanismos de control, asociado con el tiempo de rotación. Estos resultados servirán de referentes para futuras investigaciones, considerando otros factores de la conducción de inventarios en las empresas comercializadoras, que permitan diseñar sistemas de control contable para contribuir a optimizar los costos de comercialización de este sector, resaltando la necesidad del uso crítico y reflexivo de la teoría contable a lo largo del proceso investigativo, para presentar el siguiente trabajo.

\begin{abstract}
In the competitive world in which business operates, it is necessary to develop internal control mechanisms that allow companies to reduce their costs to obtain greater utility. The present work has the objective of analyzing the control of inventories, contextualized in the sector of pharmaceutical distribution of the province of GuayasEcuador, in order to determine their incidence in the costs and the profitability of the companies.

The methodology to follow in order to carry out an in-depth accounting diagnosis is based on scientific methods and techniques (observation, interviews and surveys) applied in a pharmaceutical distribution company, in the field of inventory management, being this exploratory and descriptive research. The results obtained, present the main drawbacks of inventory control in pharmaceutical distribution, which focus on the lack of control mechanisms, associated with the time of rotation. These results will serve as reference points for future research, considering other factors of the conduction of inventories in marketing companies, which allow the design of accounting control systems to contribute to optimize the marketing costs of this sector, highlighting the need for critical and reflexive use of Accounting theory throughout the investigative process.
\end{abstract}

\section{Palabras clave | keywords}

Control, costos, distribución, farmacéutica, inventarios, rentabilidad.

Control, cost, inventories, distribution, pharmaceutical, profitability 


\section{Introducción}

Es indiscutible la importancia que tiene el control de inventarios como cuenta primordial de cualquier tipo de empresa comercial, puesto que la rotación de estos se convierte en dinero en efectivo de una manera inmediata, una vez que se ha concretado la venta del mismo. Las distribuidoras farmacéuticas manejan gran cantidad de inventarios, entre los cuales se encuentran medicamentos que deben estar almacenados de manera organizada en ambientes adecuados para su conservación, colocados o embalados de manera específica, lo que hace necesario establecer controles adecuados mediante políticas, procesos y funciones, que conlleven a una adecuada organización y control de inventarios en sus bodegas. Sin embargo, la desorganización y la carencia de mecanismos de control sobre los inventarios dentro de la empresa, generan errores de control en el corto y mediano plazo, ineficiencia en el uso de recursos y reducción de la rentabilidad empresarial. Bajo este contexto, resulta interesante analizar los aspectos asociados al control de inventario que inciden en la rentabilidad de las distribuidoras farmacéuticas.

Por ello, el presente trabajo busca encontrar explicaciones a situaciones internas que afectan a la empresa, mediante la revisión de teorías y conceptos, que permitan contrastar estos conceptos contables en una realidad concreta. Para lograr el objetivo de investigación se utilizan técnicas de investigación como la entrevista y la encuesta para medir el control existente en los inventarios. Con los resultados obtenidos, se puede plantear alguna solución concreta a la problemática planteada, como el diseño de un Manual de Organización y Control de inventarios que permita organizar las gestiones de control del auditor interno en cuanto a la organización departamental en el manejo de inventarios, con sus procesos adecuados que minimicen pérdidas y garanticen los resultados fiables de los inventarios y por tanto en los estados financieros de la organización.

Un sistema de inventario es una estructura que sirve para controlar el nivel de existencia y para determinar cuánto hay que pedir de cada elemento y cuándo hay que hacerlo (Guerrero, 2009). El inventario asimismo representa la existencia de bienes almacenados destinados a realizar una operación, sea de compra, alquiler, venta, uso o transformación (Ruiz, 2011). Los inventarios representan uno de los principales recursos de que dispone una entidad comercial o industrial (Romero, 2012).

Los inventarios constituyen, en la mayoría de los casos, uno de los principales componentes del capital de trabajo de las organizaciones 
y las decisiones de inversión en este rubro. Por tanto, deberían tomarse teniendo en cuenta además del factor costo, que es el principal objetivo de los enfoques de gestión, algunos elementos asociados al riesgo y rendimiento de las mismas (Ramírez \& Manotas, 2014).

Dentro de toda organización es de vital importancia la compra y venta de bienes o servicios; de aquí la importancia del manejo del inventario (Sánchez, Vargas, Reyes \& Vidal, 2011). Bienes que están disponibles para la producción o venta o consumo interno (Zapata, 2011). Un inventario es definible como aquel conjunto de bienes, tanto muebles como inmuebles, con los que cuenta una empresa para comerciar (Barzallo, \& Solórzano, 2013).

Por lo general, los inventarios son el mayor activo dentro de los balances de las empresas. Estos contienen a todos los artículos o mercancías que dispone la empresa para su comercialización, permitiendo la compra-venta en un periodo económico determinado. Durán (2012) y Aguilar-Santamaría (2012) reconocen al inventario como un amortiguador entre dos sistemas: uno de oferta (producción o abastecimiento) y otro de demanda (clientes o distribuidores). Con estas características, se puede entender que el tamaño del inventario va a depender del comportamiento de estos sistemas. Por lo tanto, mientras más demore la cadena de abastecimiento, mayor seria el tamaño del inventario, debido al efecto látigo de la demanda. Se debe mantener un nivel adecuado de inventario para evitar posibles costos por mantenimientos o pérdidas por deterioro por excedentes, lo cual afecta directamente la rentabilidad de la empresa. Mantener elevados niveles de inventarios implica mayor utilización de recursos financieros inmovilizados afectando el flujo de operaciones. Al contrario, mantener un nivel bajo de inventarios, provocaría que se realicen mayor cantidad de pedidos a los proveedores, además de no cubrir satisfactoriamente la demanda, ocasionando pérdida de clientes, disminución de ventas y afectación en las utilidades (Durán, 2012).

Actualmente los sistemas de inventarios probabilísticos están dentro de dos grandes clasificaciones: sistemas con revisión continua y con revisión periódica (Gutiérrez, Panteleeva, Hurtado \& González, 2013).

La aplicación de un Sistema de Gestión de Inventarios es una de las alternativas más influyentes en el esfuerzo por reducir los costos y mejorar la eficiencia económica (Pérez, Cifuentes, Vásquez \& Ocampo 2012). Así, la gestión de los inventarios debe tener en cuenta los aspectos organizacionales y los actores que la afectan (López \& Gómez, 2013). 
Los almacenes, Bodegas y los Centros de Distribución constituyen un factor clave de éxito para la gestión efectiva de la cadena de abastecimientos y distribución de las organizaciones (Mora, 2011). En este sentido, la gestión de inventarios es fundamental, debido a características que generan problemas con dos clases de complejidad: (i) En primer lugar, se tiene la gran magnitud de artículos que comprenden las líneas comercializadas y (ii) la segunda clase, se debe a la naturaleza del sistema y a las variables externas que influyen en su comportamiento (Toro \& Bastidas, 2011). En este orden de ideas, la gestión de los inventarios se ha venido convirtiendo en uno de los grandes retos que enfrentan los directivos con respecto a la planificación y el control (Aguilar-Santamaría, 2012).

La administración de los inventarios dentro de las corporaciones es importante debido a que cumplen con vitales funciones como mantener la independencia entre operaciones, cubrir la incertidumbre de la demanda, dar flexibilidad a la programación de la producción, protección contra demoras en el tiempo de entrega del proveedor y aprovechar descuentos por parte de los proveedores (Izar y Méndez, 2013).

La teoría de inventarios tiene sus raíces en el modelo de Cantidad Económica de Pedido (conocida en inglés como Economic Order Quantity o por el acrónimo EOQ), propuesto por Harris en 1913. Actualmente dicho modelo permite obtener una buena aproximación de la política óptima de inventarios en varias situaciones de la vida real. Sin embargo, en sistemas de inventarios donde el deterioro tiene un impacto económico significativo, asumir que los productos tienen vida útil ilimitada conduce a la adopción de políticas de inventarios muy alejadas a las óptimas. La disminución de la calidad de los productos agrega una penalización adicional en el mantenimiento del inventario, y, por tanto, un desafío importante en la gestión de inventarios con productos perecederos es determinar una manera eficiente de mantener la disponibilidad de los artículos mientras que se evitan excesivas pérdidas por productos vencidos (Pérez y Torres, 2014).

Se conocen otros modelos de administración de inventarios como puntos de reorden con lotes dependientes del nivel de inventario, el cual indica que bajo revisión continua, se realiza un pedido con una variable suficiente para tener los inventarios cerca del nivel deseado. Sin embargo, es bastante probable que se tenga faltante durante el plazo de entrega del pedido. Otro modelo, como el de revisión periódica con lotes dependientes del nivel de inventario, indica que ordena una cantidad de 
abastecimiento que puede ser variable, lo cual va a depender del nivel de inventario que se tenga en almacenamiento. Este sistema tiene desventajas potenciales, como por ejemplo el exceso de inventario que se puede requerir para mantener esta política. Asimismo, al presentarse demanda estacional se aumenta la posibilidad de incurrir en faltantes cuando esta estacionalidad no es considerada dentro del periodo de revisión. La revisión periódica con puntos de reorden y lotes dependientes del nivel de inventario tiene como ventaja la posesión del costo global más económico de los sistemas anteriores. Sin embargo, se requiere mayor recurso y esfuerzo a nivel computacional (Osorio, 2013).

La clasificación de mercancías se puede establecer atendiendo a varios criterios, como lo son: el estado físico; las propiedades de durabilidad o caducidad, que influyen en su conservación y mantenimiento; el grado de peligrosidad; el grado de rotación y la función que desempeñen dentro del flujo logístico; la forma, el tamaño o la densidad (Escudero, 2014).

La gestión de los inventarios es cada vez más importante dentro de las administraciones de las empresas, pues determina el cumplimiento o fracaso de los objetivos establecidos (Toro y Bastidas, 2011). La gestión se desarrolla mediante el control de las operaciones, el conocimiento del proceso, y considerando las externalidades que pueden favorecer o perjudicar las operaciones del negocio. Por ello, el control interno se ha convertido en un elemento clave en la consecución de objetivos específicos en las empresas. Dentro de las estrategias de optimización de recursos siempre se debe contar con una debida planificación y estructuración de los procesos para el control interno, debido a que estos otorgan una seguridad razonable sobre el cumplimiento de metas y objetivos durante un periodo determinado. En la actualidad, las decisiones administrativas se basan en la información revelada por estos controles. Otro de los propósitos del control interno es minimizar las desviaciones y riesgos, permitiendo adelantarse a los hechos mediante la detección de posibles alteraciones a los procesos (Márquez, 2011). Así, un buen sistema de control permite que las organizaciones tengan mayores oportunidades de conseguir sus objetivos, jugando un papel fundamental en las finanzas de las administraciones empresariales (Chumpitaz, 2015).

La eficacia de los sistemas de control de inventarios depende de factores como la medición adecuada de los tiempos de reabastecimiento, el diseño de indicadores de eficiencia globales capaces de considerar 
todas la variables implícitas para el control de inventarios, y en especial de la implementación de métodos de pronóstico de demanda que permitan una estimación precisa de la tendencia y variabilidad de la demanda de cada uno de los productos que se mantengan en inventario -minimizando el error propio en la naturaleza de estos métodos- al tener en cuenta datos realistas sobre la demanda, la exclusión de datos atípicos y la selección adecuada del período base para el cálculo de pronósticos (Aguirre, Ardila, Figueroa y Romero, 2015).

El problema más común en las empresas es la existencia de excedentes y de faltantes. Muchos administradores consideran que es conveniente tener demasiado de lo que no se vende y muchos agotados de lo que sí tiene volumen de ventas. Lo interesante de este problema es que ocurre prácticamente en cualquier empresa del sector industrial o comercial (Vidal, Londoño y Contreras, 2011).

Las principales causas para la necesidad del mantenimiento de inventarios en cualquier empresa son el desfase existente entre la demanda de los consumidores y la producción o suministro de dichos productos y las fluctuaciones aleatorias de la demanda y de los tiempos de reposición en la cadena de suministro. Las estrategias más comunes para manejar estas fluctuaciones son el mejoramiento de la calidad de la información, el mantenimiento de inventarios de seguridad y la colaboración en la cadena de abastecimiento (Vidal, Londoño y Contreras, 2011).

Según Osorio (2013), los inventarios necesitan la presencia e implementación de una política confiable de control. Una óptima política de inventarios debe dar respuesta a las preguntas de cada cuánto debe revisarse el inventario, cuándo ordenar y cuánto ordenar, bien sea ítems de demanda independiente o dependiente. La metodología de estimación de políticas para darle respuesta a estas preguntas puede variar significativamente debido al tipo de producto y al ambiente de producción (Gutiérrez y Vidal, 2008). La elección del sistema de control depende de la complejidad del escenario de operación, el número de ítems que se necesitan controlar, el número de instalaciones donde se puede almacenar el inventario y la disponibilidad de la información en tiempo real (Osorio, 2013).

El debate acerca de la importancia del resultado global frente a la del resultado neto afecta a la decisión o no de revelar el primero en un estado contable independiente, ya que su información queda recogida dentro de las variaciones del neto presentado en el balance, y no sería 
necesario presentar información desagregada de forma separada si no fuera relevante (Arimany, Moya \& Rodríguez, 2011).

La metodología resultante del análisis cuenta con tres fases excluyentes en el análisis integral de la rentabilidad (Santiesteban, Fuentes y Leyva, 2011):

Fase I - Caracterización

Fase II - Diagnóstico

Fase III - Proyección de la solución

\section{Modelo de cálculo sugerido de compras}

Este modelo está diseñado en función de las fluctuaciones existentes en las variables de la demanda y de los tiempos de entrega, así como los niveles de servicio ofrecidos por la empresa a sus clientes (Mora \& Martiliano, 2012).

\section{Inventario relativo la contaminación atmosférica}

Para clasificar los contaminantes atmosféricos se atiende a: (i) Las características físicas y químicas del contaminante; (ii) Los efectos del contaminante; (iii) Las fuentes de producción o de emisión y; (iv) La transformación que experimentan los contaminantes.

\section{Material y métodos}

\section{Diseño de la investigación}

En la realización del análisis contable dentro de una empresa distribuidora farmacéutica de la provincia del Guayas (Ecuador), se decidió emplear un enfoque mixto (cuali-cuantitativo). Según Hernández, Fernández, \& Baptista (2010, p. 7) "el enfoque cualitativo utiliza recolección de datos sin medición numérica para descubrir o afinar preguntas de investigación en el proceso de interpretación". A través de esta aproximación, se logrará concebir las particularidades en los aspectos relevantes del control de inventarios, utilizando la técnica de la observación por medio del acercamiento directo con los empleados de la distribuidora y la entrevista estructurada con técnicas e instrumento de campo.

En tanto que el enfoque cuantitativo tiene como finalidad realizar análisis estadísticos (Hernández, Fernández, \& Baptista, 2010). Para el 
efecto, se aplicó un cuestionario de preguntas cerradas inherentes al tema investigado con la finalidad de evaluar su proceso, las mismas que fueron dirigidas a los empleados de la empresa y que permitieron comprender sobre la gestión que realiza el Departamento de Inventarios de esta distribuidora farmacéutica. Con esta metodología, se realiza la revisión y análisis teórico de las tendencias actuales del objeto de estudio (control de inventarios) y su rentabilidad empresarial, así como su comportamiento en el tiempo.

A partir de estas concepciones se realiza la exploración empírica cuali-cuantitativa de carácter no probabilístico y se obtienen los resultados del análisis con la siguiente metodología:

Figura 1. Metodología para realizar el análisis de control de inventario

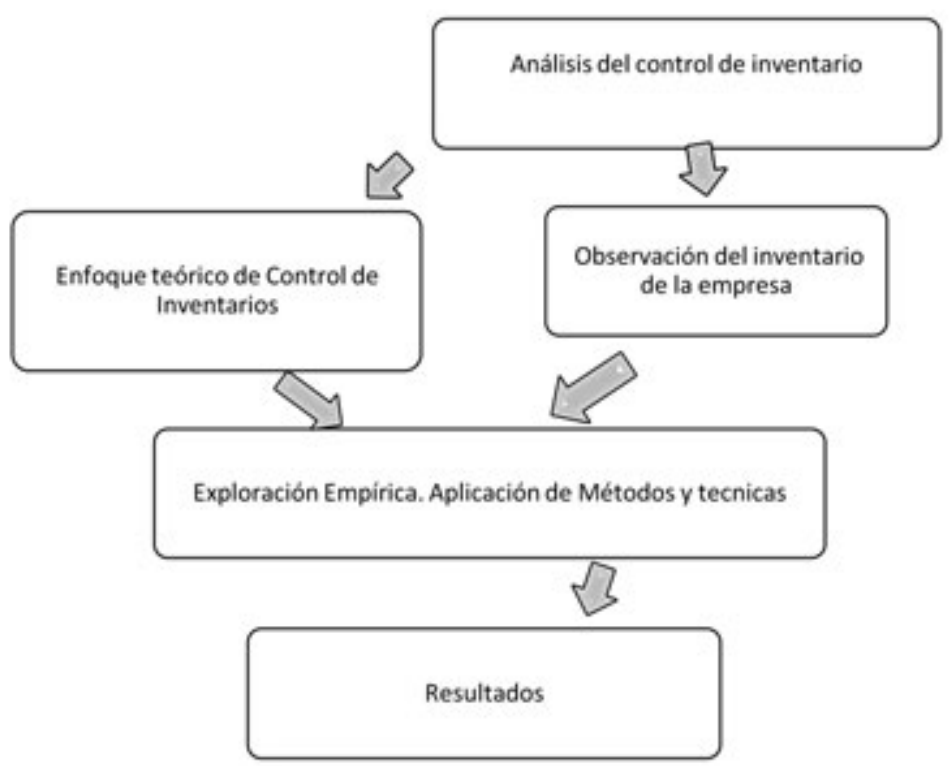

Fuente: Elaboración propia

\section{Población y muestra}

Para el desarrollo del presente trabajo, se tomó a la distribuidora farmacéutica JRC PHARMA S.A como caso de estudio. La misma cuenta con 25 empleados que laboran en diferentes áreas de la empresa. A partir 
de esta población, se determina la muestra de quince empleados relacionados al área contable y administrativa.

Castro (2003), expresa que si la población es menor a cincuenta (50) individuos, la población es igual a la muestra. En una muestra no probabilística, la elección de los miembros para el estudio dependerá de un criterio específico del investigador, lo que significa que no todos los miembros de la población tienen igualdad de oportunidad de conformarla. La forma de obtener la muestra es de este tipo intencional.

Tabla 1. Determinación de la Muestra

\begin{tabular}{|l|l|l|}
\hline \multicolumn{1}{|c|}{ Ítem } & \multicolumn{1}{|c|}{ Estrato Cantidad } \\
\hline 1 & Gerencia & 2 \\
\hline 1 & Finanzas & 3 \\
\hline 3 & Comercialización y ventas & 3 \\
\hline 4 & Compras-Importaciones & 2 \\
\hline 5 & Bodega & 5 \\
\hline & Total: Tamaño de la muestra & 15 \\
\hline
\end{tabular}

Se efectuaron entrevistas al personal que participa en el proceso de control de inventarios, para propósitos de establecer los niveles de controles y evaluaciones. Los departamentos del proceso son: finanzas, compras-importaciones, bodega y ventas (anexo 1).

Tabla 2. Preguntas para Entrevistas por departamento

\begin{tabular}{|l|l|}
\hline \multicolumn{1}{|c|}{ Preguntas por departamento } & \multicolumn{1}{|c|}{ Total } \\
\hline Dpto. de Finanzas & 5 \\
\hline Dpto. de Compras & 5 \\
\hline Dpto. de Bodega & 16 \\
\hline Dpto. de Ventas & 6 \\
\hline Total & 32 \\
\hline
\end{tabular}


La evaluación de los controles está en función a cumplimientos a la organización y a los controles de inventarios. Se establecieron cinco tipos de controles que, a criterios del investigador, se consideran necesarios para su cumplimiento, los cuales son:

Tabla 3. Evaluación departamental en control de inventarios

\begin{tabular}{|l|l|}
\hline \multicolumn{1}{|c|}{ Tipos controles } & \multicolumn{1}{c|}{ Significados } \\
\hline 1- Controles informales & Controles improvisados \\
\hline 2- Establecidos pero no escrito & Controles verbales y no escritos \\
\hline 3- Estandarizados & Controles básicos \\
\hline 4- Monitoreado & Controles escritos y monitoreados \\
\hline 5- Maximizado & Controles estrictos y monitoreados \\
\hline
\end{tabular}

Con el objetivo de poder determinar el cumplimiento en los controles, se establecieron puntajes máximos que cada área debería de cumplir para establecer su efectividad en los controles. Los puntajes máximos que las áreas deberían de cumplir una vez tabuladas las preguntas según los niveles de control que se evaluaron, se detallan en la tabla 4.

Tabla 4. Evaluación departamental en control de inventarios

\begin{tabular}{|l|l|}
\hline \multicolumn{1}{|c|}{ Evaluación Departamentos } & \multicolumn{1}{|c|}{ Puntaje Máximo } \\
\hline Dpto. de Finanzas & 25 \\
\hline Dpto. de Compras & 25 \\
\hline Dpto. de Bodega & 80 \\
\hline Dpto. de Ventas & 30 \\
\hline Total & 160 \\
\hline
\end{tabular}

Para poder determinar los puntajes máximos, se han establecido los pesos que se va a determinar como base para cada tipo de control, y se calculó un promedio de los pesos existentes (promedio 15), que servirá para determinar los puntajes que la compañía tiene con el nivel de control y organización actual. 
Tabla 5. Evaluación en pesos del nivel de control y organización

\begin{tabular}{|l|l|l|}
\multicolumn{1}{c|}{ Controles } & \multicolumn{1}{c|}{ Pesos } & \multicolumn{1}{c|}{$\%$} \\
\hline Controles informales & 5 & $7 \%$ \\
\hline Establecidos pero no escrito & 10 & $13 \%$ \\
\hline Estandarizados & 15 & $20 \%$ \\
\hline Monitoreado & 20 & $27 \%$ \\
\hline Maximizado & 25 & $33 \%$ \\
\hline Total & 75 & $100 \%$ \\
\hline
\end{tabular}

Las preguntas, una vez tabuladas con sus puntajes, serán ponderadas para establecer si los controles de la compañía son: aceptables, controles intermedios o controles deficientes. Para esto hemos considerado los niveles de cumplimientos de acuerdo al rango porcentual siguiente:

Tabla 6: Evaluación de cumplimientos

\begin{tabular}{|l|l|}
\hline \multicolumn{1}{|c|}{ Descripción de cumplimientos } & \multicolumn{1}{c|}{ Cumplimientos } \\
\hline Aceptable & $76 \%$ a $100 \%$ \\
\hline Intermedio & $51 \%$ a $75 \%$ \\
\hline Deficiente & $10 \%$ a $50 \%$ \\
\hline
\end{tabular}

Para propósitos de determinar los pesos y evaluar los niveles de cumplimientos se procede a tabular. En el conteo de las preguntas por área se procede a multiplicar por el promedio general (15) de los pesos máximos de los controles y multiplicados por el porcentaje de cada control. Esto proporcionará el peso promedio que servirá para evaluar los controles de la compañía.

Tabla 7: Total de controles por departamento

\begin{tabular}{|l|l|l|l|l|l|}
\hline \multicolumn{1}{|c|}{ Controles } & \multicolumn{5}{c|}{ Departamentos } \\
\hline & Finanzas & $\begin{array}{l}\text { Dpto. de } \\
\text { Compras }\end{array}$ & $\begin{array}{l}\text { Dpto. de } \\
\text { Bodega }\end{array}$ & $\begin{array}{c}\text { Dpto. de } \\
\text { Ventas }\end{array}$ & $\begin{array}{c}\text { Total } \\
\text { evaluación }\end{array}$ \\
\hline Controles informales & 2 & 1 & 2 & 1 & 6 \\
\hline Establecidos pero no escrito & 3 & 4 & 5 & 5 & 17 \\
\hline Estandarizados & 0 & 0 & 9 & 0 & 9 \\
\hline Monitoreado & 0 & 0 & 0 & 0 & 0 \\
\hline Maximizado & 0 & 0 & 0 & 0 & 0 \\
\hline Total & 5 & 5 & 16 & 6 & 32 \\
\hline
\end{tabular}


El siguiente cuadro presenta el resultado de esta operación, con los pesos por departamento para la determinación del cumplimiento en las evaluaciones de los controles y organización. Estas cifras se contrastarán con los valores máximos establecidos para el control de inventarios por cada área de la empresa.

Tabla 8. Peso total por control

\begin{tabular}{|l|l|l|l|l|l|}
\hline & \multicolumn{5}{|c|}{ Departamentos } \\
\hline Peso por Control & $\begin{array}{c}\text { Dpto. de } \\
\text { Finanzas }\end{array}$ & $\begin{array}{c}\text { Dpto. de } \\
\text { Compras }\end{array}$ & $\begin{array}{c}\text { Dpto. de } \\
\text { Bodega }\end{array}$ & $\begin{array}{c}\text { Dpto. de } \\
\text { Ventas }\end{array}$ & $\begin{array}{l}\text { Total peso } \\
\text { por control }\end{array}$ \\
\hline $\begin{array}{l}\text { Controles } \\
\text { informales }\end{array}$ & 2 & 1 & 2 & 1 & 6 \\
\hline $\begin{array}{l}\text { Establecidos pero } \\
\text { no escrito }\end{array}$ & 6 & 8 & 10 & 10 & 34 \\
\hline Estandarizados & 0 & 0 & 27 & 0 & 27 \\
\hline Monitoreado & 0 & 0 & 0 & 0 & 0 \\
\hline Maximizado & 0 & 0 & 0 & 0 & 0 \\
\hline Total & 8 & 9 & 39 & 11 & 67 \\
\hline
\end{tabular}

\section{Análisis y resultados}

Del total de las 32 preguntas realizadas a los entrevistados de diferentes departamentos referentes al cumplimiento del control de inventarios y que tienen como objetivo principal conocer si los controles establecidos son efectivos o deficientes, se encontraron los siguientes hallazgos:

Tabla 9: Descripción del cumplimiento por departamento

\begin{tabular}{|l|l|l|l|l|}
\hline \multicolumn{1}{|c|}{ Departamentos } & $\begin{array}{c}\text { Puntajes } \\
\text { máximos }\end{array}$ & $\begin{array}{c}\text { Puntajes } \\
\text { obtenidos }\end{array}$ & $\begin{array}{c}\text { Cumplimiento } \\
\text { de la compañía }\end{array}$ & $\begin{array}{c}\text { Descripción del } \\
\text { cumplimiento }\end{array}$ \\
\hline Dpto. de Finanzas & 25 & 8 & $32 \%$ & Deficiente \\
\hline Dpto. de Compras & 25 & 9 & $36 \%$ & Deficiente \\
\hline Dpto. de Bodega & 80 & 39 & $49 \%$ & Deficiente \\
\hline Dpto. de Ventas & 30 & 11 & $37 \%$ & Deficiente \\
\hline Total & 160 & 67 & $42 \%$ & Deficiente \\
\hline
\end{tabular}

Como se puede observar, los niveles de cumplimiento de los controles por departamento de la compañía, son deficientes y están concentrados en los controles informales y controles establecidos, pero 
no escritos, seguidos por los controles estandarizados. También se puede evidenciar, desde el punto de evaluación aplicado, que la empresa carece de los niveles de control monitoreados y maximizados.

\section{Organización, control y manejo de inventarios}

Para conocer la percepción de los empleados de la empresa referente a la organización y control de inventarios así como posibles alternativas de solución, se aplicó una encuesta a 15 empleados del área contable y financiera, cuyos resultados son los siguientes:

\section{Tabla 10. Encuesta aplicada a los empleados del área contable-financiera}

\begin{tabular}{|c|c|c|c|c|c|c|c|c|c|}
\hline \multirow[b]{2}{*}{$\mathrm{N}^{\circ}$} & \multirow[b]{2}{*}{ Preguntas } & \multicolumn{8}{|c|}{ Respuestas } \\
\hline & & Si & $\%$ & No & $\%$ & $\begin{array}{c}\text { No } \\
\text { Sabe }\end{array}$ & $\%$ & Total & $\%$ \\
\hline 1 & $\begin{array}{l}\text { ¿Considera usted que la } \\
\text { Empresa cuenta con pro- } \\
\text { cedimientos adecuados en } \\
\text { la organización y control de } \\
\text { inventarios? }\end{array}$ & 4 & $27 \%$ & 8 & $53 \%$ & 3 & $20 \%$ & 15 & $100 \%$ \\
\hline 2 & $\begin{array}{l}\text { ¿La institución cuenta con } \\
\text { diseño de procesos de control } \\
\text { de inventarios? }\end{array}$ & 2 & $13 \%$ & 8 & $53 \%$ & 5 & $33 \%$ & 15 & $100 \%$ \\
\hline 3 & $\begin{array}{l}\text { ¿Se realizan evaluaciones de } \\
\text { la organización y control de } \\
\text { inventarios para tomar desicio- } \\
\text { nes de gestión financiera? }\end{array}$ & 2 & $13 \%$ & 10 & $67 \%$ & 3 & $20 \%$ & 15 & $100 \%$ \\
\hline 4 & $\begin{array}{l}\text { ¿La gestión de requisición } \\
\text { de inventarios se genera con } \\
\text { agilidad y eficiencia? }\end{array}$ & 4 & $27 \%$ & 9 & $60 \%$ & 2 & $13 \%$ & 15 & $100 \%$ \\
\hline 5 & $\begin{array}{l}\text { ¿Considera usted que con el } \\
\text { diseño e implementación de } \\
\text { un manual de procedimientos } \\
\text { en bodega le ayudaría a mejo- } \\
\text { rar la organización y control de } \\
\text { inventarios? }\end{array}$ & 15 & $100 \%$ & 0 & $0 \%$ & 0 & $0 \%$ & 15 & $100 \%$ \\
\hline
\end{tabular}

Los resultados obtenidos en la encuesta, resaltan la carencia de organización y control de inventarios que tiene esta empresa de distribución farmacéutica, expresado en un 67\% que manifiestan tal situación. De igual manera, se evidencia la poca agilidad y eficiencia en la gestión de requisición de inventarios con un $60 \%$; así como un ineficiente manual de procesos y procedimientos para el manejo del inventario con un $53 \%$. 
Resulta interesante observar que el 100\% de la muestra indica la necesidad de contar con un manual de procedimientos para la organización y control de inventarios.

\section{Incidencia de los inventarios en las utilidades}

Para conocer la incidencia del control de inventarios en las utilidades de la empresa, se procedió a revisar la información documental contable en donde se pudo detectar que en el monto de facturación realizada en el año 2011 fue de US\$ 63000 000, vendiéndose un total de 15 033421 ítems en mercaderías, y no se encontraron reportes de cierre de inventarios de mercaderías que cuadren las entradas y salidas de mercaderías, en concordancia con lo facturado en ese periodo; por lo tanto, en ese año no se puede cuantificar pérdidas o faltantes.

En el año 2012, igualmente existe un monto en facturación de US\$ 21114 000, lo que equivale a un total de 91023423 ítems, y en la suma de los totales de salida de inventarios, no se realizó una respectiva rotación de inventarios valorada en US\$ 9235,46 , la cual se dejó entrar en fecha de caducidad a ciertos productos; generando una pérdida económica para la empresa, esto sin contar con diferencias entre la cantidad de ítems facturados y de faltantes en bodegas que se descubrieron por un total de US $\$ 1$ 030,56, los cuales pudieron ser generados por malos conteos durante la entrega de los pedidos a los clientes.

\section{Discusión y conclusiones}

Los resultados presentados reflejan las principales dificultades para el control de sus inventarios que tiene esta empresa distribuidora farmacéutica, los cuales están asociados a la poca organización departamental, carencia de flujo de procesos y control de los ítems adquiridos para la venta, así como el incumplimiento por parte del personal encargado para dicho control que permitan conducir a obtener una mejor rentabilidad empresarial. El control de inventarios debe estar enfocado a la reorganización de bodegas, mediante implantación de un sistema de control que inicie desde el momento de la requisición de un material hasta la salida del producto al mercado, pasando por los diferentes procesos logísticos de la comercialización y ordenamiento en bodega.

En concordancia con los objetivos planteados en la investigación, el presente reporte refleja los principales hallazgos obtenidos en la observación y análisis del control de inventario que incide en la rentabilidad 
empresarial contextualizado en una distribuidora farmacéutica, los cuales se centran en:

- La carencia de control presupuestario anual y mensual en los departamentos de contabilidad, finanzas, ventas y compras, no permite disponer de previsiones en la planificación de flujo de caja, rotación de los inventarios y de espacio físico para las bodegas.

- La facturación se realiza en un espacio no apropiado (bodega de los inventarios), lo que permite al personal de bodega observar los precios de los productos vendidos, que puede ocasionar conflicto de intereses, hurtos, o cualquier otra situación que afecte económicamente a la compañía.

- El escaso control interno, ya que las firmas de responsabilidad de los documentos en bodega solo cuentan con la firma del bodeguero. Estos deben contar con mínimo 3 firmas: elaborado, revisado y aprobado.

- La descoordinación entre la toma física de los inventarios mensuales y la participación del departamento contable, no permite tener certeza sobre el inventario físico y tampoco existe un informe técnico de los productos farmacéuticos que estén en cuarentena.

- La manipulación de los productos farmacéuticos requiere de un conocimiento general sobre los procesos de control de inventario por parte de las personas del personal de bodega y de los otros departamentos.

De igual manera, los resultados del presente trabajo pueden servir de referente para futuras investigaciones que permitan presentar estrategias de solución a la problemática planteada, como el diseño de un Manual de Control de Inventarios que sirva como instrumento de optimización de recursos, minimizar costos y conservación de medicamentos, insumos y productos para la venta, lo cual permitirá a la organización establecer su propio sistema de administración de inventarios para resolver de manera adecuada los aspectos relacionados con las preguntas: ¿cuánto comprar, cuándo comprar y cuánto tener?. Esta alternativa de solución y sus esquemas propuestos se visualizan en los anexos 2 y 3.

\section{Referencias}

Aguilar-Santamaría, P. A. (2012). Un modelo de clasificación de inventarios para incrementar el nivel de servicio al cliente y la rentabilidad de la empresa. Pensamiento $\mathcal{E}$ Gestión, (32), 142-164.

Aguirre-Lasprilla, S., Ardila-Rueda, W., Figueroa, L., \& Romero-Rodríguez, Daniel H. (2015). Parametrization and evaluation of Inventory Policy $(S, Q)$ in Hospi- 
tals: A case of study in Barranquilla. Prospect, 13(1), 99-105.

Arimany-Serrat, N., Moya Gutiérrez, S., \& Rodríguez Pérez, G. (2011). Relevancia valorativa del resultado global y sus componentes frente al resultado neto. Revista de Contabilidad, 171.

Barzallo Gálvez, M. J., \& Solórzano Tucunango, A. S. (2013). Análisis en el manejo del inventario y su impacto en la rentabilidad mediante la implementación de la NIC 2 en la empresa ZECANORBER S.A ubicada en el cantón Milagro en el año 2013. Universidad Estatal del Milagro, Guayas, Ecuador (Trabajo de Grado). (https://goo.gl/tpXU76).

Castro, M. (2003). El proyecto de investigación y su esquema de elaboración. (2ª .ed.). Caracas: Uyapal.

Chumpitaz, D. O. (2015). Caracterización del control interno en la gestión de las empresas comerciales del Perú 2013. In Crescendo, 6(1), 64-73.

Durán, Y. (2012). Administración del inventario. Elemento clave para la optimización de las utilidades en las empresas Visión Gerencial, 11(1), 55-78.

Escudero, M. (2014). Logística de almacenamiento. Madrid: Ediciones Paraninfo.

Guerrero, H. (2009). Inventarios: manejo y control. Ecoe Ediciones.

Gutiérrez-González, E., Panteleeva, O. V., Hurtado-Ortiz, M. F., \& González -Navarrete, C. (2013). Aplicación de un modelo de inventario con revisión periódica para la fabricación de transformadores de distribución. Ingeniería, Ingeniería Investigación y Tecnología, 14(4), 537-551.

Gutiérrez, V., \& Vidal, C. J. (2008). Modelos de gestión de inventarios en cadenas de abastecimiento: Revisión de la literatura. Revista Facultad de Ingeniería Universidad de Antioquia, (43), 134-149.

Hernández-Sampieri, R., Fernández-Collado, C., \& Baptista, M.P. (2010). Metodología de la Investigación. México: McGraw-Hill.

Izar, J. M., \& Méndez, H. (2013). Estudio comparativo de la aplicación de 6 modelos de inventarios para decidir la cantidad y el punto de reorden de un artículo. Revista Ciencia y Tecnología, (13), 217-232. DOI: http://dx.doi.org/10.18682/ cyt.v1i13.105

Márquez, G. (2011). Modelos contemporáneos de control interno. Fundamentos teóricos. Observatorio Laboral Revista Venezolana, 4(8), 115-136.

Mora, L. (2011). Gestión de Logística en centros de distribución, bodegas y almacenes. Bogotá: Ediciones Ecoe.

Mora, L., Martiliano, M. (2012). Modelos de optimización de la gestión logística. Ecoe Ediciones

Osorio, C. A. (2013). Modelos para el control de inventarios en las pymes. Panorama, 2(6), 4-10.

Pérez-Mantilla, F.A, \& Torres, F. (2014). Modelos de inventarios con productos perecederos: Revisión de literatura. Revista Ingeniería, 19(2), 9-41.

Pérez-Vergara, I., Cifuentes-Laguna, A. M., Vásquez-García, C., \& Ocampo, D.M. (2012). Un modelo de gestión de inventarios para una empresa de productos alimenticos. Ingeniería Industrial, 34(2), 227-236.

Ramírez-Reyes, G. S., \& Manotas-Duque, D. F. (2014). Modelo de medición del im- 
pacto financiero del mantenimiento de inventario de suministros. Scientia Et Technica, 19(3), 251-260. DOI: http://dx.doi.org/10.22517/23447214.8659

Romero, A. (2012). Contabilidad intermedia. México D.F: McGraw Hill.

Sánchez, M., Vargas-López, M., Reyes-Luna, B. A., \& Vidal-Vásquez, O. L. (2011). Sistema de Información para el Control de Inventarios del Almacén del ITS. Reporte de Proyecto. Conciencia Tecnológica, (41), 41-46.

Santiesteban, E., Fuentes, V., \& Leyva, E. (2011). Análisis de la rentabilidad económica: tecnología propuesta para incrementar la eficiencia empresarial. Editorial Universitaria.

Toro-Benítez, L. A., \& Bastidas-Guzmán, V. E. (2011). Metodología para el control y la gestión de inventarios en una empresa minorista de electrodoméstico. Scientia Et Technica, 16(49), 85-91.

Vidal-Holguín, C. J., Londoño-Ortega, J. C., \& Contreras-Rengifo, F. (2011). Aplicación de modelos de inventarios en una cadena de abastecimiento de productos de consumo masivo con una bodega y $\mathrm{N}$ puntos de venta. Ingeniería y Competitividad, 6(1), 10-19.

Zapata, P. (2011). Contabilidad General. Bogotá: Colombia. Gráficas de la sabana Ltda.

\section{Anexos}

\section{Anexo 1}

\section{Preguntas de entrevistas:}

\section{Área de finanzas}

1. ¿El Departamento de Finanzas posee un manual de políticas y procedimientos?

2. ¿Se efectúan presupuestos para la compra de inventarios?

3. ¿Con que periodicidad se efectúan los presupuestos para la compra de inventarios?

4. ¿La compañía dispone de créditos con los proveedores de medicamento?

5. ¿Tienen establecido un monto límite de crédito con los proveedores para las compras de inventarios?

\section{Área de compras - importaciones}

1. ¿El departamento de Compras posee un manual de políticas y procedimientos?

2. ¿Cuántas cotizaciones se solicitan a proveedores antes de decidir la adquisición de inventarios? 
3. ¿Cuál es el proceso cuando se realizan importaciones?

4. ¿Cuáles son los factores principales a la hora de decidir la compra de inventarios?

5. ¿Cuándo solicitan la compra de medicamentos se adquiere para cubrir cantidades máximas y mínimas de stock en bodega?

\section{Área de bodega}

1. ¿El departamento de Bodega posee un manual de políticas y procedimientos?

2. ¿Cuáles son los procesos ya documentados?

3. ¿Cuáles son los procesos que se encuentran en proceso de elaboración para ser documentados?

4. ¿Quién es la persona encargada de recibir los medicamentos?

5. ¿Cómo están estructuradas las bodegas?

6. ¿Cuál es la documentación que maneja el área de bodega para registrar los movimientos y/o transacciones?

7. ¿Esta documentación contiene una secuencia numérica, fecha, descripción del producto, cantidades, referencia de orden de venta, factura o cliente, que permita disponer de un control del movimiento de bodega?

8. ¿Si hubiere la documentación, existe algún grado de responsabilidad, con las debidas firmas que autorice los movimientos de los inventarios en la bodega?

9. ¿Qué tipos de documentación soporta los ingresos y egresos de bodega, en relación a las compras, importaciones, ventas, bajas, etc.?

10. ¿Bodega posee un sistema de inventarios automatizado?

11. ¿Que personal tiene acceso a este sistema de inventarios, si lo hubiere?

12. ¿En el área de bodega los usuarios del sistema tienen acceso a los costos del inventario?

13. ¿Qué sistema de costeo de inventario utilizan lo compañía?

14. ¿Con periodicidad se realizan los inventarios físicos?

15. ¿Cómo están almacenados los medicamentos en la Bodega?

16. ¿Existe un mecanismo para la salida de las muestras médicas? N/A

17. ¿En los casos de medicamentos caducados, cual es el proceso?

\section{Área de ventas}

1. ¿El departamento de Ventas posee un manual de políticas y procedimientos? 
2. ¿Cuál es el proceso de comercialización de los medicamentos?

3. ¿Quién aprueba las ventas a créditos de clientes nuevos, en relación a plazos y montos?

4. ¿Existe un documento previo a la emisión de factura, con las respectivas autorizaciones de precios y cantidades por parte de la gerencia?

5. ¿Cuál es el proceso para solicitar los medicamentos a bodega?

6. ¿Cuál es la actividad de comercialización, publicidad y/o marketing, para poder difundir los medicamentes a sus clientes?

7. ¿Cuál es el proceso para solicitar muestras médicas al área de Bodega? $\mathrm{N} / \mathrm{A}$

8. ¿Quién autoriza el despacho de muestras médicas y cuál es la cantidad límite? N/A

\section{Anexo 2. Ciclo de compras}

\begin{tabular}{|c|c|c|c|c|c|c|c|}
\hline Actrumanors & conneproves & 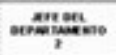 & mingen & oopen & 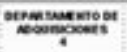 & 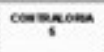 & antarat \\
\hline 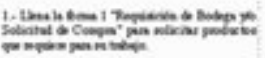 & nom & & & & & & \\
\hline 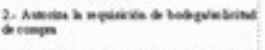 & & 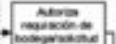 & & & & & \\
\hline 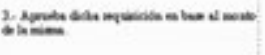 & & $\sin$ & $=$ & & & & \\
\hline 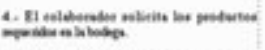 & & & & & & & \\
\hline 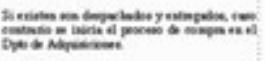 & & & & & & & \\
\hline 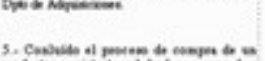 & & & & 0 & & & \\
\hline 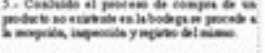 & & & & & & & \\
\hline 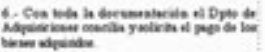 & & & & & $\cos$ & & \\
\hline 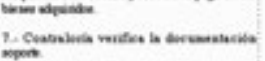 & & & & & $\lim _{0 \rightarrow \infty}$ & & \\
\hline 10.0 & & & & & & $=$ & \\
\hline 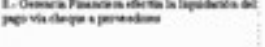 & & & & & & & 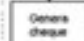 \\
\hline & & & & & & & \\
\hline & & & & & & & \\
\hline
\end{tabular}


Anexo 3. Flujograma del procedimiento en inventarios propuesto para la empresa

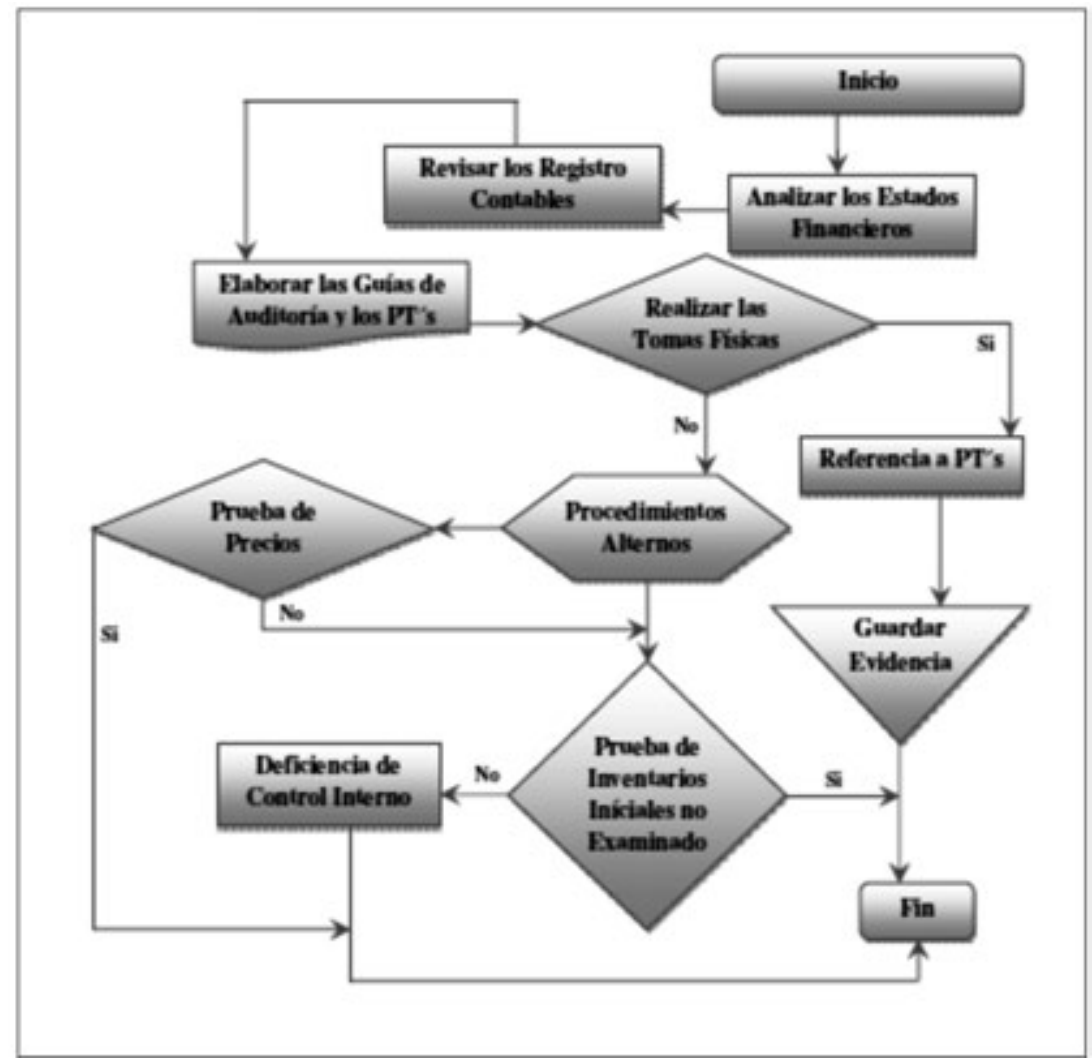

El flujograma muestra los procedimientos a seguir, de una manera muy sencilla para que la persona encargada de la revisión, no tenga inconvenientes para ejecutarlo, ya que contiene los procedimientos de auditoría necesarios, para la veracidad y la razonabilidad de los estados financieros. 